\title{
Holonomy and projective symmetry in space-times*
}

\author{
G S Hall $†$ \& D P Lonie $\ddagger$ \\ Department of Mathematical Sciences, University of Aberdeen, \\ Meston Building, Aberdeen, AB24 3UE, Scotland, U.K. \\ †e-mail: gsh@maths.abdn.ac.uk łe-mail: DLonie@aol.com
}

December 27, 2018

\begin{abstract}
It is shown using a space-time curvature classification and decomposition that for certain holonomy types of a space-time, proper projective vector fields cannot exist. Existence is confirmed, by example, for the remaining holonomy types. In all except the most general holonomy type, a local uniqueness theorem for proper projective symmetry is established.
\end{abstract}

\section{Introduction}

Some techniques for studying projective symmetry in general relativity theory were developed by the present authors in [1, 2]. That study was heavily based on the algebraic structure of the curvature tensor and included an account of the relationship between the existence of (proper) projective symmetry in a space-time and the latter's holonomy type. This account unfortunately suffered from technical restrictions on the 'rank' of the curvature tensor and these restrictions were removed in [3]. A more natural and elegant account of this relationship will be given here. To avoid undue repetition, the results in [1] will be used regularly here as will those dealing with space-time holonomy theory in [4, 5].

Throughout, $M$ will denote a (smooth) space-time manifold with (smooth) Lorentz metric $g$ and $M$ will be assumed non-flat in the sense that the associated curvature tensor (with components $R_{a b c d}$ ) does not vanish over any non-empty open subset of $M$. The tangent space to $M$ at $m \in M$ is denoted

*Submitted to Classical and Quantum Gravity, IOP publishing 
by $T_{m} M$ and a comma, a semi-colon and the symbol $\mathcal{L}$ are used for a partial, a covariant and a Lie derivative respectively. A global smooth (in fact $C^{3}$ is sufficient [1]) vector field $X$ on $M$ is called projective if its associated local diffeomorphisms (flows) map geodesics into geodesics (not necessarily preserving the affine parameter). This is equivalent to the condition that, on decomposing the covariant derivative of $X$ in any coordinate system in $M$ into its symmetric and skew-symmetric parts as $X_{a ; b}=\frac{1}{2} h_{a b}+F_{a b}$ $\left(h_{a b}=h_{b a}, h=\mathcal{L}_{X} g, F_{a b}=-F_{b a}\right), h$ satisfies [6, 7] 1]

$$
h_{a b ; c}=2 g_{a b} \psi_{c}+g_{a c} \psi_{b}+g_{b c} \psi_{a}
$$

for some uniquely determined closed 1-form $\psi$ (the projective 1-form of $X$ ) on $M$. If $h_{a b ; c}=0$ on $M$ (equivalently, $\psi=0$ on $M$ [1]), $X$ is affine (and the associated local flows also preserve the affine parameter of the geodesics of $M$ ) and, otherwise, $X$ is proper projective. If $X$ is projective and satisfies $\psi_{a ; b}=0$ on $M, X$ is called special projective.

\section{Curvature decomposition}

In this section a review is given of a convenient classification of the curvature tensor of $M[8]$. Let $m \in M$ and consider the linear map $f$ from the 6-dimensional vector space $B(m)$ of bivectors at $m$ into itself, given by

$f: F^{a b} \rightarrow R_{c d}^{a b} F^{c d}$. The curvature tensor at $m$ can be put into exactly one of the following five disjoint classes (and note that the class labelling differs from that in [1]).

Class $A$ This is the most general class and the curvature will be said to be of this class if it is not one of the classes $B, C, D$ or $O$ below.

Class $B$ The curvature is said to be of class $B$ if the range of $f$ is 2dimensional and spanned by a timelike-spacelike pair of simple bivectors with orthogonal blades.

Class $C$ The curvature is said to be of class $C$ if the range of $f$ is 2- or 3 -dimensional and if there exists $k \in T_{m} M, k \neq 0$, such that each bivector $F$ in this range satisfies $F_{a b} k^{b}=0$.

Class $D$ The curvature is said to be of class $D$ if the range of $f$ is 1 dimensional.

Class $O$ The curvature is said to be of class $O$ if it vanishes at $m$. 
The dimension of the range of $f$ is just the curvature rank when the curvature tensor $R_{a b c d}$ is written in the well-known way as a $6 \times 6$ symmetric matrix. The following results are then straightforward to check (c.f. [8]).

1. For the classes $A$ and $B$ there does not exist $k \in T_{m} M, k \neq 0$, such that $F_{a b} k^{b}=0$ for each $F$ in the range of $f$.

2. For class $A, \operatorname{dim}($ range of $f) \geq 2$ and if $\operatorname{dim}($ range of $f) \geq 4$ the class is necessarily $A$.

3. The vector $k$ in the definition of class $C$ is unique up to a scaling.

4. For class $D$ the identity $R_{a[b c d]}=0$ shows that the range of $f$ is spanned by a simple bivector.

Let $V_{m}$ be a subspace of $B(m)$. If $F \in V_{m}$ is simple, its blade is a 2dimensional subspace of $T_{m} M$. If $F \in V_{m}$ is not simple it may be written as $F=G+H$ where $G$ and $H$ are a timelike-spacelike pair of simple bivectors with orthogonal blades which are uniquely determined by $F[9]$. Now define the subspace $U_{m} \subseteq T_{m} M$ associated with $V_{m}$ as the span of the union of the blades of the members of $V_{m}$ (including both blades for a non-simple member of $\left.V_{m}\right)$. Thus if $V_{m}$ is non-trivial, $\operatorname{dim} U_{m} \geq 2$, if $V_{m}$ contains a non-simple member $\operatorname{dim} U_{m}=4$ and if $\operatorname{dim} U_{m}<4$ each member of $V_{m}$ is simple and there exists $k \in T_{m} M, k \neq 0$, such that $F_{a b} k^{b}=0$ for each $F$ in $V_{m}$. Also, if all members of $V_{m}$ are simple, $\operatorname{dim} V_{m} \leq 3$. To see this, briefly, suppose $\operatorname{dim} V_{m} \geq 4$ with all members of $V_{m}$ simple. Let $F$ and $G$ be independent members of $V_{m}$ so that $F, G$ and $F+\lambda G$ are simple for each $\lambda \in \mathrm{R}$. Now $E \in V_{m}$ is simple if and only if $E^{a}{ }_{b}{ }^{*} E^{b}{ }_{c}=0$, where ${ }^{*}$ denotes the duality operator. Applying this to the above three bivectors yields $F_{b}^{a *} G^{b}{ }_{c}+G^{a}{ }_{b}{ }^{*} F_{c}^{b}=0$. Now, since $F$ is simple, there exists $k \in T_{m} M$, $k \neq 0$, such that $F_{a b} k^{b}=0$ and a contraction of the previous equation with $k_{a}$ gives $\left(k_{a} G_{b}^{a}\right)^{*} F_{c}^{b}=0$. Thus the vector $G_{b}^{a} k^{b}$, which is in the blade of $G$, is either zero or orthogonal to the blade of the (necessarily simple) bivector ${ }^{*} F$ and hence in the blade of $F$. In either case, the blades of $F$ and $G$ (and of ${ }^{*} F$ and ${ }^{*} G$ ) intersect non-trivially and so there exists $k^{\prime} \in T_{m} M, k^{\prime} \neq 0$, such that $F_{a b} k^{\prime b}=G_{a b} k^{\prime b}=0$. Thus, adopting a standard notation in which a simple bivector whose blade is spanned by $r, s \in T_{m} M$ is written $r \wedge s$, one has $p, x, y \in T_{m} M$ such that $F=p \wedge x$ and $G=p \wedge y$. Now let $H \in V_{m}$ with $F, G$ and $H$ independent and apply the above argument to the pairs $(F, H)$ and $(G, H)$ to see that either $H=p \wedge z\left(z \in T_{m} M\right)$ or that the above vectors $x$ and $y$ may be chosen so that $H=x \wedge y$. Finally, introduce $K \in V_{m}$ with $F, G, H$ and $K$ independent and apply a similar argument to $F, G$ and $K$ 
to see that one contradicts either the independence of $F, G, H$ and $K$ or the fact that all members of $V_{m}$ are simple. This completes the proof. It follows from the first part of this proof that if all members of $V_{m}$ are simple and $\operatorname{dim} V_{m}=2$, there exists $k \in T_{m} M, k \neq 0$, such that $F_{a b} k^{b}=0$ for each $F$ in $V_{m}$, and $\operatorname{dim} U_{m}=3$.

Now identify the range of $f$ with $V_{m}$ above. Then, if the curvature class at $m$ is $D, \operatorname{dim} V_{m}=1$ and $\operatorname{dim} U_{m}=2$ whereas if it is $C, \operatorname{dim} V_{m}=2$ or 3 and $\operatorname{dim} U_{m}=3$ and for class $B$ one has $\operatorname{dim} V_{m}=2$ and $\operatorname{dim} U_{m}=4$. If $\operatorname{dim} U_{m} \leq 3$ the curvature class at $m$ is $O, C$ or $D$. It can also now be checked by using 'rank type' arguments that there exists an open neighbourhood $W$ of $m$ such that $\operatorname{dim} V_{m^{\prime}} \geq \operatorname{dim} V_{m}$ and $\operatorname{dim} U_{m^{\prime}} \geq \operatorname{dim} U_{m}$ for each $m^{\prime} \in W$.

Denote by the same symbols $A, B, C, D$ and $O$ the subsets of $M$ consisting of precisely those points where the curvature is of that class. Then $M=A \cup B \cup C \cup D \cup O$ and, since $M$ is non-flat, $O$ has empty interior in the usual manifold topology on $M$. The following theorem can now be proved (and an independent proof can be found in [10]).

Theorem $1 M$ may be disjointly decomposed as

$$
M=A \cup \operatorname{int} B \cup \operatorname{int} C \cup \operatorname{int} D \cup Z
$$

where int denotes the topological interior operator in $M, A$ is open, $Z$ is a closed subset of $M$ defined by the disjointness of the decomposition and where $\operatorname{int} Z=\emptyset$ (and so $M \backslash Z$ is an open dense subset of $M$ ).

Proof. This will be briefly sketched making free use of the above comments together with the usual rank theorems. Let $m \in A$ so that $\operatorname{dim} V_{m} \geq 2$ and $\operatorname{dim} U_{m}=4$. Then there exists an open neighbourhood $N_{1}$ of $m$ such that $\operatorname{dim} U_{m^{\prime}}=4$ for each $m^{\prime} \in N_{1}$ and so the curvature class at each point of $N_{1}$ is $A$ or $B$. If $\operatorname{dim} V_{m} \geq 3$, one may choose $N_{1}$ such that $\operatorname{dim} V_{m^{\prime}} \geq 3$ for each $m^{\prime} \in N$ and so $N_{1} \subseteq A$. If $\operatorname{dim} V_{m}=2$, and since (from remarks above because $m \in A$ ) $V_{m}$ must contain a non-simple member, it may be arranged that $V_{m}$ is spanned by non-simple bivectors $F$ and $G$. But the blade-pairs of $F$ and $G$ cannot coincide at $m$ (since then $m \in B$ ) and so they will not coincide over some open neighbourhood $N_{2}$ of $m$. Then with the original $N_{1} \subseteq A \cup B, N_{1} \cap N_{2} \subseteq A$ and $A$ is open. Now let $m \in B$ so that $V_{m}$ contains a non-simple member. Then $V_{m^{\prime}}$ contains a non-simple member for each $m^{\prime}$ in some neighbourhood $N_{3}$ of $m$ and so $N_{3} \subseteq A \cup B$ and so $A \cup B$ is open. A simple consideration of rank on $V_{m}$ shows that $A \cup B \cup C$ and $A \cup B \cup C \cup D$ are also open. Finally, to show $\operatorname{int} Z=\emptyset$, let $W \subseteq Z$ be open. Then, by disjointness, $W \cap A=\emptyset$. Then, since, $A \cup B$ is 
open, $W \cap B=W \cap(A \cup B)$ is open. But $Z$ is disjoint from $\operatorname{int} B$ and so $W \cap B=\emptyset$. Similarly, $W \cap C=W \cap D=W \cap O=\emptyset$ and so $W=\emptyset$ and $\operatorname{int} Z=\emptyset$.

\section{Holonomy structure}

A holonomy classification scheme for a space-time $M$ was given in [4, 5]. There it was described how, when $M$ is simply connected, the holonomy group of $M$ (which is a connected Lie group) is determined by its Lie algebra, this latter being a subalgebra of the Lie algebra of the Lorentz group. The holonomy type was then labelled $R_{1}, \ldots, R_{15}$ following a similar labelling of the subalgebras of the Lorentz algebra given in [1] with $R_{1}$ being the trivial case when $M$ is flat and $R_{15}$ the most general. It is noted here for future reference that the dimensions of the non-trivial holonomy algebras are $R_{2}$, $R_{3}, R_{4}$ (dimension 1), $R_{6}, R_{7}, R_{8}$ (dimension 2), $R_{9}, R_{10}, R_{11}, R_{12}, R_{13}$ (dimension 3), $R_{14}$ (dimension 4 ) and $R_{15}$ (dimension 6 ). The type $R_{5}$ cannot occur as the holonomy group of a space-time (see, e.g. 4]). Another useful result (from infinitesimal holonomy theory) is that, in the notation of the last section, the range of $f$ at each $m \in M$ is in an obvious sense, using the bivector representation of the Lorentz algebra, contained in the holonomy algebra [12. Thus if the holonomy type of $M$ is $R_{1}$, the curvature class at any point of $M$ is $O$ whereas for types $R_{2}, R_{3}$ and $R_{4}$ it is $O$ or $D$, for $R_{6}$ and $R_{8}$ it is $O, D$ or $C$, for $R_{7}$ it is $O, D$ or $B$, for $R_{9}$ and $R_{12}$ it is $O, D, C$ or $A$, for $R_{10}, R_{11}$ and $R_{13}$ it is $O, D$ or $C$ and for $R_{14}$ and $R_{15}$ it could be any curvature class. A table which gives, for each holonomy type, the covariantly constant and recurrent vector fields admitted by $M$ can be found in [5].

In [1] some results regarding the incompatibility of the existence of proper projective vector fields on $M$ and certain holonomy types for $M$, together with the assumed constancy of the dimension of the range of the linear map $f$, were given. In the next section these assumptions will be removed with the help of theorem 1. The role played by the map $f$ is also crucial and, in particular, the interplay between its range and kernel where the latter consists of those bivectors $G$ satisfying $R_{a b c d} G^{c d}=0$.

\section{The main theorems}

Suppose $X$ is a projective vector field on $M$ as in section 1. Suppose also that some bivector $G$ lies in the kernel of the map $f$. Then it was shown in 
[1] that the following relations hold;

$$
\begin{gathered}
h_{a e} R_{b c d}^{e}+h_{b e} R_{a c d}^{e}=g_{a c} \psi_{b ; d}-g_{a d} \psi_{b ; c}+g_{b c} \psi_{a ; d}-g_{b d} \psi_{a ; c} \\
\psi_{a ; c} G_{b}^{c}+\psi_{b ; c} G_{a}^{c}=0
\end{gathered}
$$

These two equations dovetail in the following way. Condition (3) at $m \in M$ is equivalent to the statement that, if $G$ is simple, all non-zero vectors in the blade of $G$ are eigenvectors of $\psi_{a ; b}$ with equal eigenvalue, and if $G$ is non-simple, the same condition holds for each of its (uniquely determined orthogonal pair of) blades, but where the eigenvalue may depend on the blade [8]. Thus, if the kernel of $f$ is 'sufficiently' large, each member of $T_{m} M$ will be an eigenvector of $\psi_{a ; b}$ with the same eigenvalue and so, at $m$, $\psi_{a ; b}=\alpha g_{a b}(\alpha \in \mathbf{R})$. But this last condition on $\psi_{a ; b}$ is equivalent to the right hand side of (2) vanishing and hence to the vanishing of the left hand side of (2). Finally, the vanishing of the left hand side of (2) is the condition that (loosely stated) $h$ may be an 'alternative metric' [8, 13] and its solution for $h$ given the range of the map $f$ is known [8, 14. In particular, if the range of $f$ satisfies the class $A$ condition at $m, h$ is proportional to $g$ at $m$. Using techniques such as these it was shown in [1] that if $X$ is projective on $M$, the algebraic structure of the curvature tensor at points of the subsets $B, C$ and $D$ of $M$ resulted in the projective 1 -form $\psi$ vanishing on $\operatorname{int} B$ and $\operatorname{int} C$ and satisfying $\psi_{a ; b}=0$ on int $D$. These results will be useful in proving the next two theorems.

Theorem 2 Let $M$ be a non-flat simply connected space-time of holonomy type $R_{2}, R_{3}, R_{4}, R_{6}, R_{7}, R_{8}$ or $R_{12}$. Then $M$ does not admit a proper projective vector field.

Proof. Let $X$ be a projective vector field on $M$ and let $M$ be decomposed as in theorem 1. If $\operatorname{int} B \neq \emptyset$ let $m \in \operatorname{int} B$ and let $W \subseteq \operatorname{int} B$ be an open, connected, simply connected neighbourhood of $m$. Regarding $W$ as a spacetime with metric given by the restriction of $g$ to $W$, it follows (as remarked above) from theorem 5 in [1] that $\psi=0$ on $W$ and hence on $\operatorname{int} B$. Similarly, theorem 4 in [1] shows that, if $\operatorname{int} C \neq \emptyset, \psi=0$ on $\operatorname{int} C$. Now let $m \in A$. The assumption in the statement of the theorem regarding the possible holonomy types of $M$ and the remarks at the end of the first paragraph of section 3 show that $\operatorname{dim}($ range of $f) \leq 3$ and hence that the kernel of $f$ has dimension at least three. The same assumption and the fact that the range of $f$ is 'contained' (in an obvious sense) in the holonomy algebra enables the kernel of $f$ to be calculated at $m$. This calculation easily reveals that this kernel is sufficient to establish, using (3) as described above, that $\psi_{a ; b}=\gamma g_{a b}$ for 
some function $\gamma: A \rightarrow \mathbf{R}$. Thus, as explained at the beginning of this section, the right and hence left hand side of (2) is zero and the vanishing of the left hand side shows that $h$ is proportional to $g$ at $m$ and hence that, on $A, h_{a b}=\beta g_{a b}$ for some function $\beta: A \rightarrow \mathbf{R}$. A substitution of this into (1) and comparing a contraction of (1) with $g^{a b}$ to a contraction with $g^{a c}$ then shows that $\psi=0$ on $A$. Now let $m \in \operatorname{int} D$ and with $W \subseteq \operatorname{int} D$ as above. Then it follows (theorems 7 and 9 in [1) that, if $X$ is proper, the holonomy type of $W$ is $R_{10}, R_{11}$ or $R_{13}$ and a contradiction follows since these can not be contained in the assumed holonomy groups for $M$. Hence $\psi=0$ on $\operatorname{int} D$. Thus $\psi=0$ on the open dense subset $M \backslash Z$ of $M$ and hence on $M$ and the result follows.

Theorem 3 Let $M$ be a non-flat simply connected space-time which admits a proper projective vector field $X$. Then $M$ has holonomy type $R_{10}, R_{11}$ or $R_{13}$ if and only if $X$ is special projective.

Proof. If $M$ has any of these holonomy types and since the range of $f$ at any $m \in M$ is contained in the holonomy algebra, it follows from result (i) following the classification in section 2 that the subsets $A$ and $B$ in the decomposition of $M$ in theorem 1 are empty. Thus $M=C \cup \operatorname{int} D \cup Z$ (since, from rank considerations, $C$ is now open). If $X$ is a proper projective vector field on $M$, it follows from theorem 4 in [1] that $\psi=0$ on $C$ and from theorem 3 in [1] that $\psi_{a ; b}=0$ on $\operatorname{int} D$. Thus $\psi_{a ; b}=0$ on the open dense subset $M \backslash Z$ of $M$ and hence on $M$ and so $X$ is special projective. [Also, since $X$ is proper and $\psi$ covariantly constant, $\psi$ cannot be zero at any $m \in M$ and so $C=\emptyset$ and $M=D \cup Z$ with $D$ open in $M$.] Conversely, if $M$ admits a proper special projective vector field $X$, then from section 7 in [1] one has a decomposition $M=D \cup Z$ and theorem 6 in [1] shows that $M$ admits a single independent covariantly constant vector field (represented by $\psi^{a}$ ). From the possible holonomy types available in this case, and recalling the exclusions of theorem 2 , it follows that the holonomy type of $M$ is $R_{10}, R_{11}$ or $R_{13}$.

If the conditions and conclusions of theorem 3 hold, the local form of the metric in $M \backslash Z$ is known [1. Thus theorems 2 and 3 essentially complete the study of (proper) projective symmetry in space-times except in the cases of holonomy type $R_{9}, R_{14}$ and $R_{15}$. 


\section{$5 \quad$ Space-times of holonomy type $R_{9}$ and $R_{14}$}

A consideration of space-times of non-zero constant curvature [15] and certain F.R.W. models [16] show that proper projective vector fields exist in holonomy type $R_{15}$ space-times. In this section a brief discussion of spacetimes of holonomy type $R_{9}$ and $R_{14}$ will be presented and which will reveal a local uniqueness result for and the existence of proper projective symmetry.

Let $M$ be a simply-connected space-time of holonomy type $R_{9}$ or $R_{14}$ and let $X$ be a proper projective vector field on $M$ with associated 1-form $\psi$. Then $M$ admits a global, null, nowhere-zero recurrent vector field $l$ (i.e. in each local coordinate domain of $M, l_{a ; b}=l_{a} p_{b}$ for some global covector field $p)$. On differentiating this relation and using the Ricci identity one finds $l_{d} R_{a b c}^{d}=l_{a} G_{b c}$, where $G_{a b}=2 p_{[a ; b]}$, and then the identity $R_{[b c d]}^{a}=0$ leads to $G_{[a b} l_{c]}$ and hence to $G_{a b} l^{b}=\gamma l_{a}$ for some function $\gamma: M \rightarrow \mathbf{R}$. Now let $p \in M$ and $U$ an open neighbourhood of $p$ on which $l$ has been extended to a smooth null tetrad $l, n, x, y$ (whose only non-vanishing inner products are $\left.l^{a} n_{a}=x^{a} x_{a}=y^{a} y_{a}=1\right)$. The fact that the range of $f$ must be contained in the holonomy algebra means, from the holonomy algebras in the $R_{9}$ and $R_{14}$ cases [1], that, in $U$, this range must be a subspace of the span of the bivectors $l \wedge n, l \wedge x, l \wedge y$ and $x \wedge y$ in the $R_{14}$ case and of the span of the

first three of these in the $R_{9}$ case. Thus if $H$ is either of the bivectors $l \wedge x$ and $l \wedge y, R_{a b c d} H^{c d}=0$ and a contraction of (2) with $H^{c d}=0$ gives

$$
H_{a}^{d} \psi_{b ; d}+H_{b}{ }^{d} \psi_{a ; d}=0
$$

It follows from [8] that $l, x$ and $y$ are eigenvectors of $\psi_{a ; b}$ at each $p \in U$ with equal eigenvalue and hence that, on $U$,

$$
\psi_{a ; b}=\alpha g_{a b}+\beta l_{a} l_{b}
$$

for smooth functions $\alpha$ and $\beta$ on $U$. Next one differentiates (5) and uses the Ricci identity $2 \psi_{a ; b c]}=\psi_{d} R_{a b c}^{d}$ to get

$$
\psi_{d} R_{a b c}^{d}=2 g_{a[b} \alpha_{, c]}+2 l_{a}\left(l_{[b} \beta_{, c]}+2 l_{[b} p_{c]}\right)
$$

A contraction of (6) with $l^{b}$ then gives, on $U$,

$$
G_{d a} \psi^{d} l_{c}=l_{a} \alpha_{, c}-\left(\alpha_{, b} l^{b}\right) g_{a c}-\left(\beta, b l^{b}+2 \beta p_{b} l^{b}\right) l_{a} l_{c}
$$

A contraction of (7) with $x^{a} x^{c}$ then gives $\alpha_{, b} l^{b}=0$ and a back substitution into (7) followed by a contraction with $n^{a}$ reveals that $\alpha_{, c}$ is proportional to $l_{c}$ on $U$. A contraction of (6) with $l^{a}$ then gives $\left(\psi_{d} l^{d}\right) G_{b c}=0$ on $U$. 
Now consider the open subset $A \subseteq M$ in the decomposition of theorem 1 and in which the results of the previous paragraph hold. In addition, one now has (result (i) in section 2) that $G$ is nowhere zero on $A$ and so $\psi_{a} l^{a}=0$ on $A$. Thus $\left(\psi_{a} l^{a}\right)_{; b}=0$ and use of the recurrence condition on $l$ together with (5) then gives $\alpha=0$ and so $\psi_{a ; b}=\beta l_{a} l_{b}$ on $A$. [From this it can also be shown that $\psi$ is proportional to $l$ on $A$ but this fact will not be required.] Now let $X$ and $Y$ be projective vector fields on $M$ with associated 1-forms $\psi$ and $\phi$ and with (1) also holding with $h$ and $\psi$ for $X$ replaced with $H$ and $\phi$ for $Y$. Then, on $A, \psi_{a ; b}=\beta l_{a} l_{b}$ and $\phi_{a ; b}=\delta l_{a} l_{b}$ for smooth functions $\beta$ and $\delta$ on $A$ and on writing down (2) for $X$ and $Y$ one easily sees that the associated right hand sides are proportional and hence

$$
\tilde{h}_{e a} R_{b c d}^{e}+\tilde{h}_{e b} R_{a c d}^{e}=0 \quad(\tilde{h}=\delta h-\beta H)
$$

Now it follows from [8, 14] that, on $A$, the only solutions to (8) are of the form $\tilde{h}_{a b}=\nu g_{a b}$ for some smooth function $\nu$ on $A$ and hence that $\delta h_{a b}-\beta H_{a b}=\nu g_{a b}$. Now let $V \subseteq A$ be the subset of $\mathrm{A}$ on which $\beta$ and $\delta$ vanish. If $\operatorname{int} V \neq \emptyset$ (and note that since $A$ is open in $M$, it makes no difference whether the interior is taken in $M$ or in the subspace topology in $A$ ) then $\psi_{a ; b}=0$ and $\psi_{d} R_{a b c}^{d}=0$ and so $\psi_{a} \equiv 0$ on int $V$ (result (i) section 2 again). Similar remarks apply to $\phi_{a}$ and so $X$ and $Y$ are affine on int $V$. Now consider the open subset $W \equiv A \backslash V$ of $M$. If $p \in W$ then at least one of $\beta$ and $\delta$ is non-zero at $p$ and hence in some open connected neighbourhood $U(\subseteq W)$ of $p$. Suppose it is $\delta$ (a similar argument applies if it is $\beta$ ) so that, on $U$, one may write $h=\rho H+\sigma g$ for smooth functions $\rho$ and $\sigma$ on $U$. Then (1) gives on $U$

$$
2 g_{a b} \chi_{c}+g_{a c} \chi_{b}+g_{b c} \chi_{a}=H_{a b} \rho_{, c}+g_{a b} \sigma_{, c} \quad(\chi=\psi-\rho \phi)
$$

Then, at any $q \in U$, contract (9) with a non-zero vector $r^{c}$ chosen to be orthogonal to $\chi_{c}, \rho_{, c}$ and $\sigma_{, c}$, giving $2 r_{(a} \chi_{b)}=0$, and hence $\chi_{c}=0$ at $q \in U$. It follows that $\chi_{a}$ vanishes on $U$. So let $E \subseteq U$ be the open subset of $U$ (and of $M$ ) on which $\rho_{, c}$ does not vanish. Then (9) shows that, on $E, H_{a b}=\mu g_{a b}$ for some smooth function $\mu$ on $E$ and so, from (1) applied to $H$ and $\phi$, one easily finds that if $E \neq \emptyset, \phi_{a}$ and hence $\phi_{a ; b}$ vanish on $E$. This contradicts the fact that $\delta$ never vanishes on $U$ and so $E=\emptyset$. So $\rho_{, a}$ vanishes on $U$ ( $\Rightarrow \rho$ is constant on $U$ since $U$ is connected) and then $Z \equiv X-\rho Y$ is affine on $U$ since it is projective on $U$ with vanishing projective 1 -form $\chi$.

To summarise the previous paragraph, given any point $p$ in the open dense set int $V \cup W$ of $A$ there exists an open neighbourhood of $p$ in which some linear combination of $X$ and $Y$ is affine. [In fact, since one is working on 
a subset of $A$, it is known that proper affine vector fields cannot be admitted on (any open subset of) this subset and hence this linear combination of $X$ and $Y$ must be homothetic.] Also the restrictions of $X$ and $Y$ to $\operatorname{int} B$ and int $C$ in the decomposition of $M$ in theorem 1 are necessarily affine (as remarked in the proofs of theorems 2 and 3) and for each $p \in \operatorname{int} D$ there is a connected open neighbourhood $U$ of $p$ on which some linear combination of $X$ and $Y$ is affine [1. Thus, in the notation of the previous paragraph one may disjointly decompose $A$ as $A=W \cup \operatorname{int} V \cup Z^{\prime}$ where $Z^{\prime}$ is defined by the disjointness of the decomposition and is easily shown to have empty interior in the subspace topology on $A$ from $M$ and hence in the topology of $M, \operatorname{int} Z^{\prime}=\emptyset$. Then one has the disjoint decomposition $M=W \cup \operatorname{int} V \cup$ $\operatorname{int} B \cup \operatorname{int} C \cup \operatorname{int} D \cup Z \cup Z^{\prime}$ and some elementary topology reveals that $Z \cup Z^{\prime}$ is closed with empty interior and so $\tilde{M} \equiv W \cup \operatorname{int} V \cup \operatorname{int} B \cup \operatorname{int} C \cup \operatorname{int} D$ is open and dense in $M$. One then has the following local uniqueness result.

Theorem 4 Let $M$ be a non-flat simply connected space-time of holonomy type $R_{9}$ or $R_{14}$. Then if $X$ and $Y$ are projective vector fields on $M$ there exists an open dense subset $\tilde{M}$ of $M$ such that each $p \in \tilde{M}$ admits an open neighbourhood $U$ such that some linear combination of the restrictions of $X$ and $Y$ to $U$ is affine on $U$.

Consider the metric given in a global chart domain $M$ with coordinates $\left(x^{1}, x^{2}, x^{3}, x^{4}\right)=(u, v, x, y)$ with $0<u, v<\infty,-\infty<x, y<\infty$, by

$$
d s^{2}=2 d u d v+\sqrt{u v^{-3}} d v^{2}+v^{2} e^{g(x, y)}\left(d x^{2}+d y^{2}\right)
$$

for some smooth function $g$ on $\mathbf{R}^{2}$. The only non-vanishing components (up to algebraic symmetries) of the curvature tensor are

$$
R_{1212}=\frac{1}{8 w^{3}} \quad R_{2323}=R_{2424}=\frac{-e^{g(x, y)}}{4 w} \quad R_{3434}=-\frac{v^{2}}{2} e^{g(x, y)} \nabla^{2} g
$$

where $w=\sqrt{u v}$ and $\nabla^{2} g \equiv \frac{\partial^{2} g}{\partial x^{2}}+\frac{\partial^{2} g}{\partial y^{2}}$. The vector field $l$ on $M$ defined by $l^{a}=(1,0,0,0)$ is null and recurrent on $M$ and, since it is easily checked from (11) that $R_{a b c d} l^{d}$ never vanishes on $M, l$ is not covariantly constant over any non-empty subset of $M$. The existence of such a vector field $l$ together with the facts that the rank of the bivector map $f$ at $p \in M$ is either 4 or 3 (the latter occurring if and only if $\left.\left(\nabla^{2} g\right)_{p}=0\right)$ and that at each $p \in M$ the range of $f$ can be spanned by simple bivectors shows that the holonomy type of the metric (10) is either $R_{9}$ or $R_{14}[5]$. It is clearly type $R_{14}$ if $f$ has rank 4 at some point of $M$ and, less obviously, type $R_{9}$ if $\nabla^{2} g$ vanishes identically on 
$M$. To see this last result one notes that if $\nabla^{2} g \equiv 0$ on $M$, and with $p \in M$ fixed, the range of $f$ at any $p^{\prime} \in M$ is spanned by the bivectors $\frac{\partial}{\partial u} \wedge \frac{\partial}{\partial x}$, $\frac{\partial}{\partial u} \wedge \frac{\partial}{\partial y}$ and $\frac{\partial}{\partial u} \wedge \frac{\partial}{\partial v}$ (and $l=\frac{\partial}{\partial u}$ ) at $p^{\prime}$. On parallel transport of these bivectors from $p^{\prime}$ along some curve $c$ to $p$ the bivectors accumulated at $p$, for all points $p^{\prime}$ and all associated curves $c$, span the holonomy algebra of $M$ by the Ambrose-Singer theorem [17] (see also [5]). Since $l$ is recurrent (and thus its direction is unchanged under parallel transport) and since the null and simple properties of bivectors are also, respectively, unchanged under parallel transport, it is easily checked that the set of bivectors accumulated at $p$ is the span of the above set of three bivectors at $p$ and so the holonomy type is $R_{9}$. Finally, the vector field $X$ given by $X^{a}=\left(u v, v^{2}, 0,0\right)$ can now be checked to be a proper projective vector field on $M$ with associated 1form $\psi$ given by $\psi=(0,1,0,0)$ independently of the rank of $f$ and thus confirms the existence of proper projective vector fields in both $R_{9}$ and $R_{14}$ holonomy types.

\section{References}

[1] Hall G S and Lonie D P 1995 Class. Quant. Grav. 12 1007-1020

[2] Hall G S 1996 Proc. 6th Int. Conf. on Diff. Geom. and its Appl., Brno, Czech Republic Ed. J. Janyska, I. Kolár and J. Slovák (Masaryk University, Brno) 567-571.

[3] Hall G S 2002 unpublished

[4] Hall G S 1993 Proc. 5th Int. Conf. on Diff. Geom. and its Appl., Opava, Czech Republic Ed. O. Kowalski and D. Krupka (Silesian University, Opava) 201-210.

[5] Hall G S and Lonie D P 2000 Class. Quant. Grav. 17 1369-1382

[6] Eisenhart L P 1966 Riemannian Geometry (Princeton)

[7] Katzin G H, Levine J and Davis W R 1969 J. Math. Phys. 10 617-629

[8] Hall G S and McIntosh C B G 1983 Int. J. Theor. Phys. 22 469-476

[9] Sachs R K 1961 Proc. Roy. Soc. A264 309-337

[10] Patel M T 2004 Ph.D. Thesis submitted to the University of Aberdeen

[11] Schell J F 1961 J. Math. Phys. 2 202-206 
[12] Kobayashi S and Nomizu K 1963 Foundations of Differential Geometry: Vol.1 (Interscience, New York)

[13] Ihrig E 1975 Int. J. Theor. Phys. 1423

[14] Hall G S 1983 Gen. Rel. Grav. 15 581-589

[15] Barnes A 1993 Class. Quant. Grav. 10 1139-1146

[16] Hall G S 2000 Class. Quant. Grav. 17 4637-4644

[17] Ambrose W and Singer I M 1953 Trans. Amer. Math. Soc. 75 428-443 